\title{
Reciclaje de plásticos de consumo masivo. Caso comunidad de reciclaje Nashira
}

\author{
Orozco-Echeverri, Lina Marcela ${ }^{a}$; Neira-Liscano, Henry Sneyder ${ }^{\mathrm{b}}$; Ramirez-Triana, Nelida \\ Yaneth $^{\mathrm{c}}$. \\ ${ }^{a}$ Universidad Nacional de Colombia, Colombia.1morozcoe@unal.edu.co. \\ ${ }^{\mathrm{b}}$ Universidad Nacional de Colombia, Colombia. hsneiral@unal.edu.co. \\ ${ }^{\mathrm{c}}$ Universidad Nacional de Colombia, Colombia.nyramirezt@unal.edu.co.
}

\section{Resumen}

Desde sus orígenes los plásticos han revolucionado el mundo debido a su versatilidad frente a otros materiales, sin embargo, el volumen de material ha crecido ostensiblemente como se registra en los datos de Plastics - the facts 2015. An analysis of European plastics production, demand and waste data (2015), donde se afirma que 311 millones de toneladas de plástico se han producido en el mundo en el año 2014.

De otra parte, según el informe del Banco Internacional de Desarrollo (2011), el negocio del reciclaje en Latinoamérica, es en su mayoría informal. En Colombia, los recicladores desempeñan una labor ambiental importante siendo ellos quienes recolectan el $60.9 \%$ de los residuos reciclables, y obtienen una remuneración muy baja (aun cuando manipulan materias primas con alto valor económico), siendo sus ingresos mensuales en promedio 22,14 USD es decir menos de un dólar diario, lo cual, enmarca esta población en índices de pobreza extrema, lo cual establece una situación necesario de intervención con el fin de dignificar el trabajo y erradicar la pobreza extrema entrando en consonancia con objetivos a nivel mundial (Programa de Naciones Unidas, 2015).

Con estos elementos preliminares del volumen de materiales plásticos y el trabajo subvalorado de los recicladores, este proyecto propone reciclar plásticos desarrollando una extrusora que produzca la transformación del material plástico de consumo masivo desechado, en filamento para impresión 3D, -teniendo en cuenta la emergente masificación de las impresoras 3D FDM (Fused Deposition Material).

Este trabajo es desarrollado en conjunto con una comunidad de "reciclaje Nashira" en el Bolo San Isidro, corregimiento del Valle del Cauca - Colombia y plantea su desarrollo a través de metodologías de co-diseño incluyendo a la comunidad activamente en el proyecto (Sanders \& Stappers, 2014), a su vez es necesario abordar los elementos claves de este proceso mediante estrategias sistémicas (Hernandis, 2015), asi a partir de este enfoque se define la configuración del objeto final con el diseño como medio transformador de aspectos intangibles como el conocimiento, asociado una dimensión sociocultural donde la interacción es dada siempre por la comunidad como actor principal.

Palabras clave: Diseño social, Co-diseño, Reciclaje de plástico, Modelado sistémico, Extrusora de filamento. 


\begin{abstract}
Since its origins the plastics have revolutionized the world due to its versatility to other materials, however, the volume of material has grown ostensibly as recorded on data from Plastics - the facts 2015. An analysis of European plastics production, demand and waste data (2015), where it is stated that 311 million tons of plastic were produced worldwide in 2014.

Furthermore, according to the report of the International Development Bank (2011), the recycling business in Latin America, is mostly informal. In Colombia, recyclers play an important environmental work being they who collected $60.9 \%$ of recyclable waste, and earn very low wages (even when handling raw materials with high economic value), and its monthly income on average \$22.14 USD less than a dollar a day, which frame this population in extreme poverty rates, which establishes a necessary intervention situation in order to dignify the work and eradicate extreme poverty coming into line with global targets (United Nations Program, 2015).

With these preliminary elements of the volume of plastic materials and recyclers underrated work, this project proposes developing a recycle plastics extruder to produce plastics processing consumer material discarded in filament for $3 D$ printing, taking into account the emerging massification 3D FDM (Fused Deposition Material) printers. This work is developed in conjunction with a community of "recycling Nashira" in Bolo San Isidro, municipality of Valle del Cauca - Colombia and raises its development through methodologies co-design including the community actively in the project (Sanders \& Stappers, 2014), in turn is necessary to address the key elements of this process by systemic strategies (Hernandis, 2015) and from this approach, the configuration of the final object is defined by the design as a medium transformer of intangibles such as knowledge associated to sociocultural dimension where interaction is always given by the community as the main actor.
\end{abstract}

Keywords: social design, Co-design, recycling plastic, systemic modeling, Extruder filament.

\title{
1. Introducción
}

Este proyecto se desarrolla desde el diseño industrial planteado como una actividad sistémica que permite identificar las necesidades y controlar los procesos que consiguen desarrollar el producto (Hernandis \& Valenzuela, 2014), se aborda el estudio de caso de la comunidad Nashira, (única formalmente constituida en la ciudad de Palmira) que lleva a cabo la actividad del reciclaje, siendo esta, una labor importante tanto para el medio ambiente como para la sociedad (Berenguer \& Corraliza , 2000). Esta investigación es abordada desde dos enfoques; el primero, consiste en el trabajo conjunto comunidad - diseñadores y el segundo desde un abordaje sistémico. Desde el primer enfoque, la experiencia de la comunidad y el acompañamiento constante del equipo de diseño, permiten el acercamiento al contexto para determinar las condiciones geográficas, económicas y culturales, a partir de la metodología de co-diseño (Sanders \& Stappers, 2014), que establece este reconocimiento para analizar la situación desde diferentes 
perspectivas. El segundo enfoque, establece los aspectos técnicos a través del modelado sistémico de producto (Hernandis \& Iribarren, 2000) y se identifican de manera clara cada uno de los requerimientos de los sistemas de la propuesta objetual, basada en los insumos obtenidos de la primera fase con el grupo de reciclaje. Los dos enfoques concretan una respuesta a la problemática, surge de las ideas de la comunidad quienes al estar involucrados en el desarrollo del proyecto y crean apropiación de los resultados; el modelado sistémico, faculta el diseño, la construcción e implementación de una extrusora de filamento para impresión 3D a partir de material plástico reciclable, que permite la transformación del plástico recolectado en una materia prima que puede ser comercializado a un mejor precio del que reciben actualmente por el plástico.

\section{Justificación}

\subsection{Social}

Actualmente en Colombia el reciclaje de plásticos es una labor realizada en su mayoría por recicladores informales, y según el BID (2011) son ellos quienes hacen el mayor aporte a la recolección del material reciclable con una cifra que alcanza el $60,9 \%$ del plástico recolectado a nivel nacional. Sin embargo, dicha labor establece una remuneración tan baja que apunta a los índices de pobreza extrema con ingresos de menos de dos dólares diarios ${ }^{14}$, aun cuando la industria del plástico reconoce el potencial económico del material plástico reciclable asegurando pérdidas de entre 80.000 y 120.000 millones de dólares cada año por envoltorios plásticos no reutilizados (Ellen Macarthur foundation, 2016).

La fabricación de filamento plástico (para impresión 3D) a partir del material reciclado por comunidades vulnerables por pobreza, crea espacios de trabajo que contribuyen al mejoramiento de la calidad de vida, a partir de mayor remuneración económica obtenida por su trabajo. Este trabajo se justifica desde lo social teniendo en cuenta las condiciones del contexto en el cual es desarrollada la labor.

\subsection{Ambiental}

Se resalta la importancia y la necesidad de reciclar, dado que la masificación del plástico en el mundo se ha convertido en una problemática cada vez más difícil de controlar, como lo advierten los estudios presentados en el foro económico mundial (The new plastics economy: rethinking the future of plastic, 2016), que indican que de seguir sin tomar acciones, para el año 2050 el peso de los residuos plásticos será superior al peso de todos los peces en el planeta, por tanto es imperiosamente necesario el proponer soluciones desde diferentes perspectivas para prestar atención a las alarmas y contribuir con la mejora de esta situación ambiental. Brindar una materia prima a partir del producto reciclado de desechos plásticos como PET o HDPE, que finalmente terminan en depósitos o rellenos sanitarios donde pueden tardar más de 150 años en descomponerse, justifica este trabajo desde lo ambiental.

\section{Caso estudio comunidad de reciclaje Nashira}

Ubicada en el corregimiento El Bolo, en la ciudad de Palmira, Departamento del Valle del Cauca, en Colombia, Nashira es una comunidad de reciclaje rural conformada por mujeres en su mayoría madres cabeza de hogar, quienes en la labor del reciclaje encuentran una alternativa para suplir sus necesidades económicas, y a su vez contribuyen al mejoramiento de las condiciones ambientales de la eco aldea a

\footnotetext{
${ }^{14}$ Tasa de incidencia de la pobreza sobre las bases de $\$ 1.90$ dolares por día (PPA). Tomado del banco mundial y comisión económica para américa latina y el caribe - CEPAL
} 
través de proyectos de formación y capacitación de separación en la fuente, recolección y manejo de residuos sólidos.

Su labor parte del aprendizaje empírico por lo cual está mujeres muestran un gran interés y dedican parte de a su tiempo a aprender cada día más sobre los materiales que recolectan, su debido tratamiento y cómo disponer adecuadamente de ellos, esta situación derivó en acercamientos y diálogos con profesionales, entre ellos los diseñadores.

Sin embargo, la ausencia de conocimiento e información en el negocio del reciclaje, se ha visto reflejada en las ganancias obtenidas; los ingresos recibidos por la comercialización del material recolectado son muy bajos, no cuentan con la experiencia, el recurso suficiente o el conocimiento técnico necesario para realizar procesos de transformación de los residuos que pudieran dar valor agregado al material.

Por ello este trabajo se encuentra oportuno para brindar herramientas que ayuden a la consolidación de los procesos de reciclaje, haciendo uso de herramientas metodológicas de diseño participativo (Sanders, 2013) para desarrollar capacitaciones específicas y plantear la implementación de transformación del material recolectado para aumentar las ganancias, es así como se plantea la construcción de una extrusora (para filamento de impresión 3D) en un proceso de construcción que involucra a la comunidad de forma activa, que contribuye al mejoramiento de su calidad de vida y que dignifica la labor tan importante que realizan en cada jornada de recolección siempre en búsqueda del bienestar humano (Ramirez, Cardozo , \& Lecuona, 2012).

\section{Metodología}

Se trabaja en esencia con 2 metodologías de diseño para lograr el cumplimiento de los objetivos, en una primera instancia se trabaja bajo los parámetros definidos por Sanders (Sanders \& Stappers, 2014), haciendo uso de metodologías de co-diseño, esta metodología sugiere el abordaje con la comunidad y el trabajo con la misma, permitiendo el desarrollo de talleres que ayudan al reconocimiento de las materias primas recolectadas y a la capacitación, comprensión desarrollo y apropiación de la máquina extrusora.

Posteriormente se aplican metodologías de una adaptación de los autores al modelo planteado por Hernandis (Hernandis \& Iribarren, 2000) en cuanto se refiere a sus modelados de producto y empresa, junto con metodología de caja negra expuesta por (Cross, 2003). Este enfoque permite visualizar desde lo general -la comunidad-, el planteamiento de un modelo, que debe ser rentable y sostenible y desde lo particular el desarrollo de un producto para transformar el material plástico recolectado.

\subsection{Workshops y trabajo con la comunidad}

Bajo el planteamiento de workshops, se logró el acercamiento con la comunidad en dos oportunidades, la primera "workshop de materiales" (Fig. 1) fue la apertura de espacios de capacitación sobre características de materiales, usos convencionales y métodos de fácil reconocimiento y clasificación, del cual se obtuvieron paneles gráficos construidos en conjunto, con los cuales pudieran interactuar y que albergaran la información requerida por el grupo en el momento de realizar las pruebas las cuales fueron aplicadas a probetas de tres materiales (PET.HDPE, y PP) y llevadas a cabo por primera vez en compañía de las cinco integrantes del grupo de reciclaje en una sesión de tres horas, donde las pruebas que se usaron para el reconocimiento de los materiales consistieron en exposición al fuego directo mediante el uso de un encendedor, siendo los resultados a tener en cuenta el color y la forma de la llama, el color del humo y el color del goteo del material; adicionalmente se realizó una prueba de densidad en agua, de la cual los resultados posibles consistían en si la probeta permanecía en la superficie del agua o por el contrario llegaba al fondo del recipiente. 

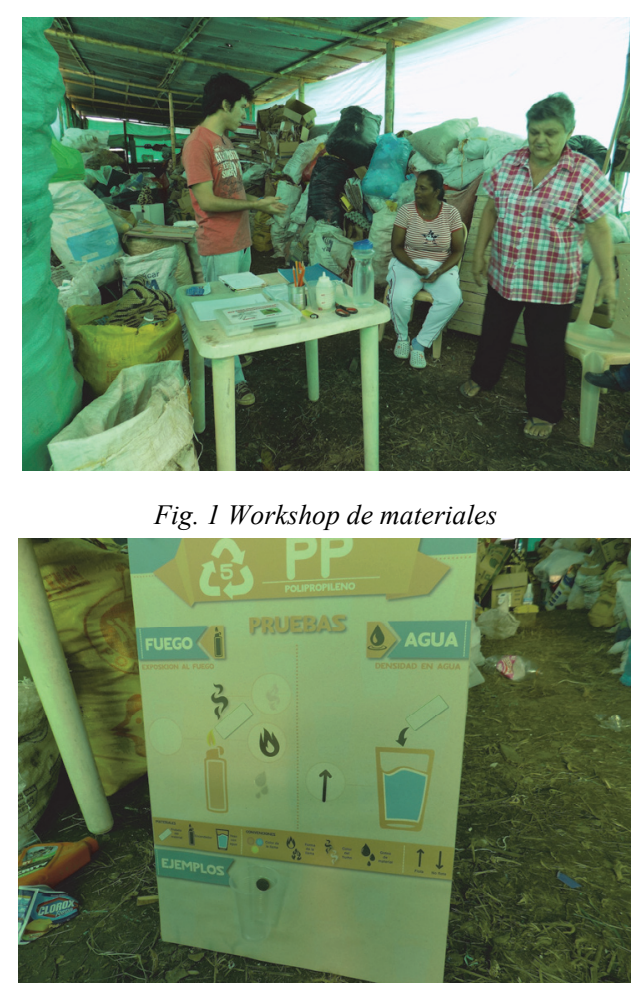

Fig. 2 Formato y diagramación del panel

Para este desarrollo se estableció que la construcción de elemento gráfico daría pautas básicas sobre los materiales y las pruebas, por lo que el equipo de diseño preestableció el formato y la diagramación (Fig. 2) de los tres paneles diferenciados entre ellos por medio de colores, dejando los espacios pertinentes para que las integrantes de la comunidad fueran construyéndolos haciendo uso de adhesivos que contenían la información y los resultados de las pruebas en los materiales, a medida que estas se realizaban.

Posteriormente se llevó a cabo el segundo ejercicio con la comunidad "workshop de formas básicas", con el objetivo de llegar a propuestas formales a partir de la construcción de volúmenes básicos que pudieran ser reinterpretados como componentes del sistema de extrusión, cuya finalidad fue incentivar la participación de las integrantes en el proceso de configuración morfológica de la máquina, promoviendo la apropiación por el proyecto con el planteamiento de ideas propias y aumentando las expectativas sobre el resultado formal con la reinterpretación de sus propuestas, por lo que para este taller se propuso llevar a cabo la actividad en 4 partes (Tabla 1) en una sesión de tres horas de trabajo.

Tabla 1. Contenidos de las actividades. 


\begin{tabular}{|c|c|}
\hline ACTIVIDAD & CONTENIDO \\
\hline Contextualización & $\begin{array}{r}\text { Procesos de reciclaje actuales, uso de maquinarias y } \\
\text { alternativas de uso del filamento plástico. }\end{array}$ \\
\hline Explicación & $\begin{array}{r}\text { Funcionamiento general de máquinas de extrusión, otros } \\
\text { procesos de transformación y alternativas mecánicas para } \\
\text { procesar materia prima. }\end{array}$ \\
\hline Construcción & $\begin{array}{r}\text { Elaboración de modelos con formas básicas como } \\
\text { reinterpretación de las partes de la extrusora. }\end{array}$ \\
\hline $\begin{array}{c}\text { Socialización de } \\
\text { resultados }\end{array}$ & Muestra de resultados en grupo y retroalimentación del \\
proceso para aclarar posibles dudas.
\end{tabular}

1. Contextualización: en esta primera etapa se realiza una explicación sobre los procesos actuales de reciclaje, posibles métodos y maquinaria usada a nivel industrial de los cuales el grupo no tuviese conocimiento y finalmente la posible disposición o uso que podría tener el filamento fabricado.

2. Explicación: para llevar a cabo el taller se realizó una explicación introductoria al proceso de extrusión del plástico, maquinarias usadas, y principios básicos de funcionamiento con el fin de brindar la información que fuera necesaria y pertinente para esta etapa del proceso.

3. Construcción: se construye con el grupo un modelo representativo de una máquina extrusora, donde se identificaron sus partes y el orden en que el sistema debía ser construido, teniendo en cuenta algunas propuesta que surgieron del grupo en cuanto a la disposición de elementos como la tolva, respecto al tornillo de extrusión y su forma.

4. Socialización de resultados: en un escenario de participación y discusión, algunas integrantes del grupo dieron sus aportes hacia el modelo construido, proponiendo cambios en la forma y brindando alternativas de solución a partes técnicas del sistema a medida que se socializaban los resultados de la actividad.

En este workshop se evidenció la motivación por el proceso de capacitación (Fig. 3) y la apropiación de la información brindada en las explicaciones y charlas realizadas desde los primeros encuentros; con esta información el grupo realizó un modelo representativo (Fig. 4) y expuso sus ideas con las demás integrantes sobre la interpretación del proceso de extrusión.

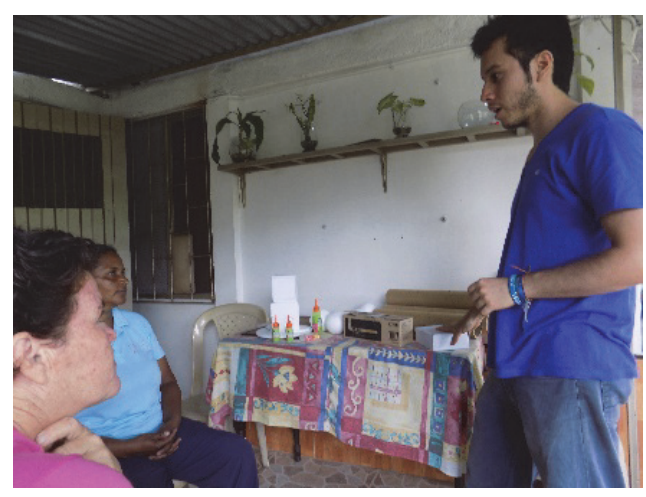

Fig.3 Capacitación sobre el proceso de extrusión. 


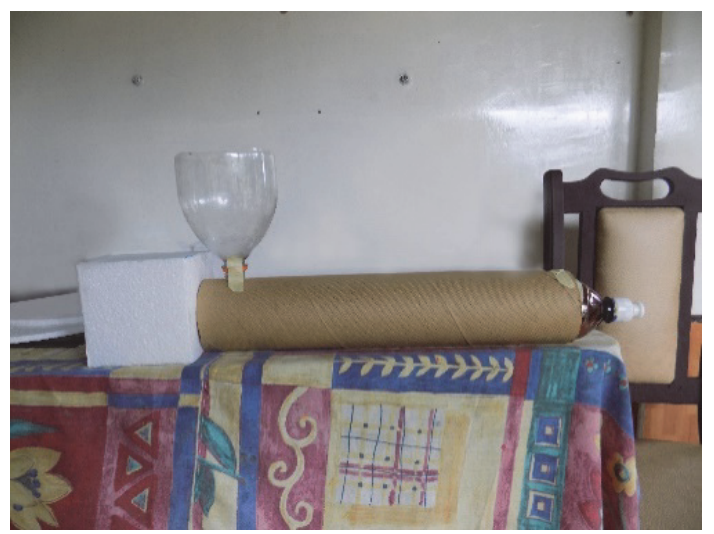

Fig.4 Construcción de modelo representativo.

\subsection{Modelado del sistema}

Paralelo a este proceso se inició el análisis de la máquina desde la perspectiva sistémica, estableciendo inicialmente los sistemas y subsistema existentes como flujos de información a través de una "caja negra" (Cross, 2003) que descompone la función principal en funciones secundarias y esenciales con sus respectivas variables de entrada y salida (Fig. 5 y Fig. 6).

Acto seguido, frente al abordaje del análisis preliminar del diseño de una extrusora se decidió escalar ampliamente y se optó por ampliar el estudio a procesos previos a la extrusión, teniendo en cuenta las variables que podían incidir en la toma de decisiones sobre la construcción o implementación de algunos procedimientos como lavado y peletizado, que pudiesen complementar la producción del filamento, entendiendo las posibilidades resultantes como modelos de negocio alternativos a la venta de la materia prima.

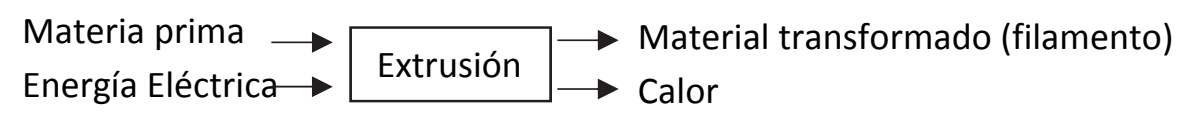

Fig.5 Modelo de sistemas de la "Caja negra” elaborada para el sistema de extrusión. 


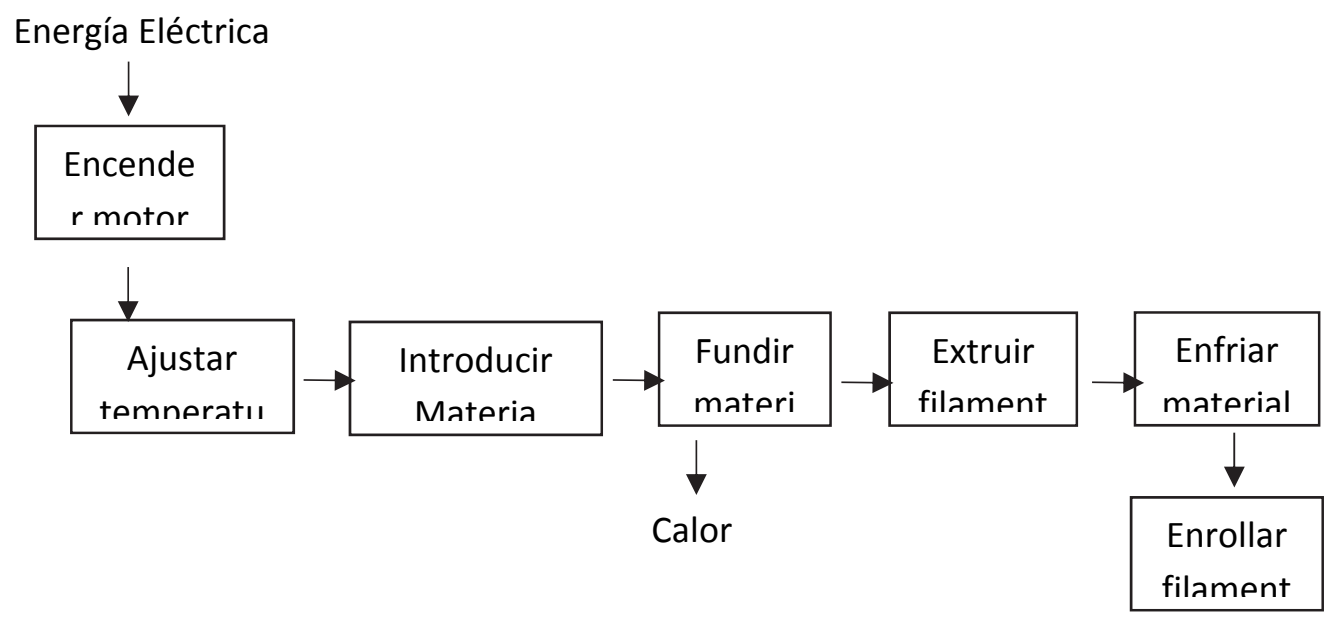

Fig.6 Descomposición de funciones para el sistema de extrusión.

Dado que el sistema de extrusión estaba comprendido por un número reducido de pasos generales, se aplicaron conceptos de escalabilidad que permitieron establecer la necesidad de procesos previos para el debido tratamiento de la materia prima, entendido desde la recolección del material reciclable, hasta el almacenamiento del producto final siendo la extrusión un subsistema del proceso de reciclaje del plástico, esto con el fin de identificar aquellos pasos que pudieran ser llevados a cabo por la misma comunidad sin necesitar otros recursos, especialmente aquellos que significaran una inversión o gasto elevado como energía eléctrica para el funcionamiento de máquinas adicionales o agua para el lavado y enfriado del material, de esta manera se propuso un sistema de producción en línea que tuviese en cuenta los procesos como son llevados a cabo actualmente (Fig. 8). Para esto se desarrolló un esquema sinóptico (Fig. 8) que tuviese en cuenta las posibilidades de comercializar el material en mejores condiciones, eliminando la dificultad de almacenamiento que representaba el no estar peletizado ni pesado y que tuviese en cuenta la propuesta de fabricación de filamento plástico tanto para venta, como para usos alternativos que la misma comunidad pudiese plantear.

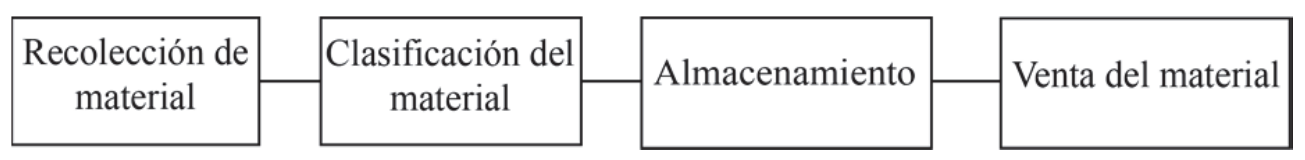

Fig. 7 proceso actual de reciclaje.

En este análisis, las variables tenidas en cuenta para la toma de decisiones sobre la disposición del material se basaron en dos aspectos, el primero que consiste en la forma de cómo se comercializa el material reciclable actualmente, donde el precio de venta se define según el peso sin los procesos de prensado ni lavado y por otro lado, la alternativa de producción de filamento con el material reciclable una vez que este que cumpla las características necesarias; en este caso en el proceso actual se identificaron actividades que podrían convertirse en cuellos de botella por la falta de espacio para el almacenamiento como es el caso del material que es lavado y almacenado sin ser peletizado, el cual ocupa grandes cantidades en volumen, pero NO en peso, lo que se traduce a poca ganancia. Por ello, se 
replanteo el esquema de producción modificando el orden de las actividades actuales con sus correspondientes variables, adicional a esto, el proceso de secado que no es llevado a cabo por su complejidad y que sería solucionado realizando el peletizado previamente (Cuadro de modificaciones en la figura.8).

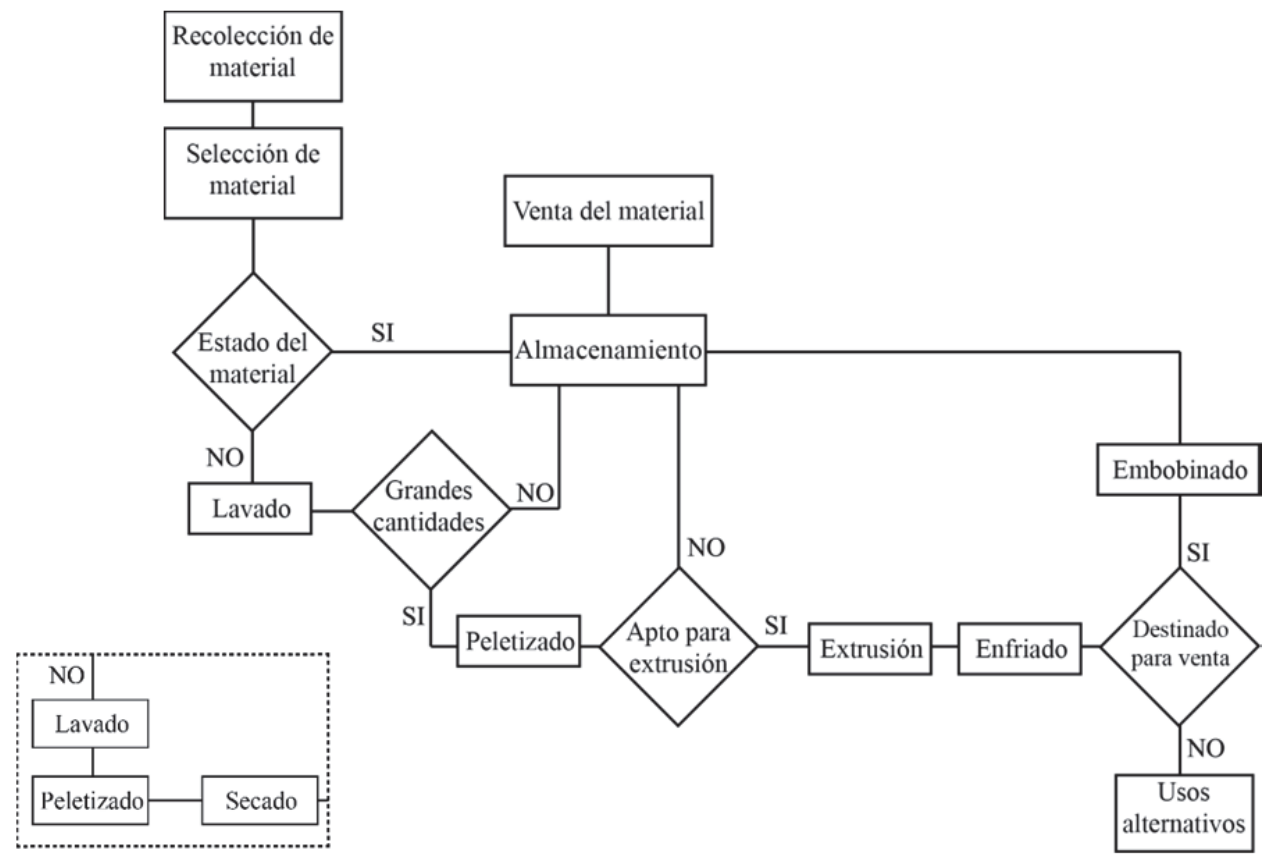

Fig.8 Esquema sinóptico para la fabricación de filamento-en línea discontinua las modificaciones posteriores.

A partir de este procedimiento se identificaron aspectos técnicos, que contribuyeron a la fijación de requerimientos, los cuales fueron relacionados directamente con un sistema funcional que permitiera solucionar una problemática específica organizadas bajo criterios de orden jerárquico de acuerdo al momento en el que son llevadas a cabo en el proceso de transformación, entendiendo que para comprender la estructura se deben examinar tanto su composición a nivel interno como las funciones que desempeña, su relación con el entorno y cada uno de los sistemas específicos con los que interactúa y que a su vez componen la función global.

\subsection{Desarrollo de la propuesta}

Una vez llevado a cabo el proceso de análisis de las condiciones de la comunidad se dio inicio a la construcción de la maquina extrusora, para esto se tuvieron en cuenta dos aspectos, el primero fueron las propuestas resultantes de los dos workshop realizados con la comunidad, con el fin de llegar a una configuración formal diferente a los existentes, y la segunda basada en los aspectos técnicos que constituyen el proceso de extrusión dividido en subsistemas funcionales y los elementos que corresponden a cada uno (fig. 10). 


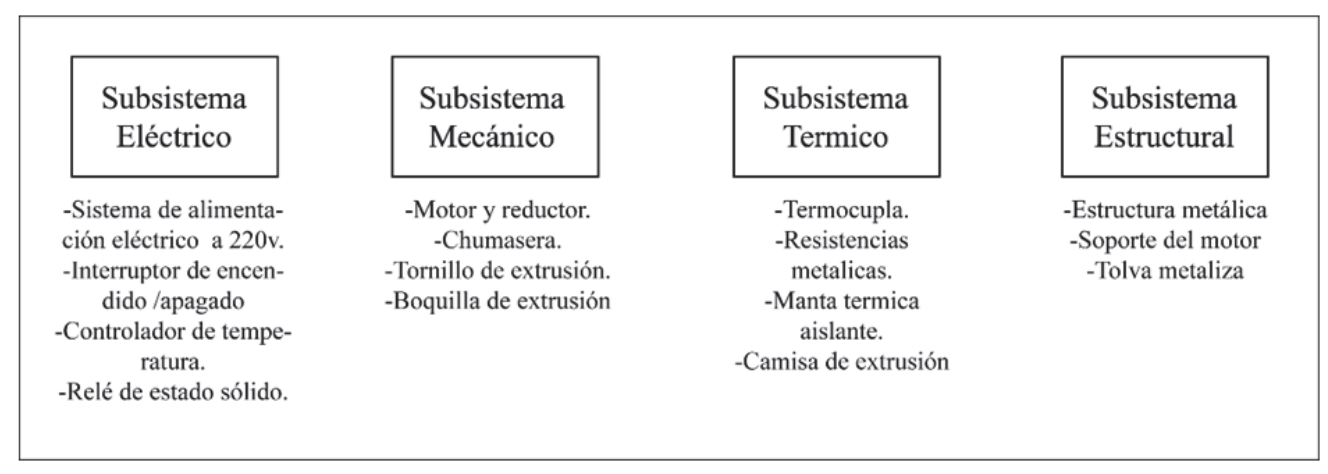

Fig.9 Subsistemas de la máquina de extrusión.

De esta manera se establecieron los requerimientos de la máquina y así mismo se adquirieron las partes según las especificaciones necesarias. Realizado dicho proceso se analizaron los elementos de la máquina en los cuales se podía intervenir de acuerdo a las apreciaciones hechas por la comunidad en espacios de participación anteriores, siendo las propuestas aplicadas a los subsistemas a través de colores que permitieran identificar de manera fácil cada uno de los elementos de la maquina (fig.10); haciendo uso de esta información, se determinó la posibilidad de usar múltiples colores para definir cada una de las partes de manera que comunicara el cuidado que se debía tener en las zonas de algunos sistemas, como lo es el subsistema térmico donde se manejan temperaturas elevadas, zonas de alimentación como la tolva en caso de atascamiento de material o mal funcionamiento del motor por posibles fallas en el sistema de alimentación eléctrica o conexiones en los componentes electrónicos.

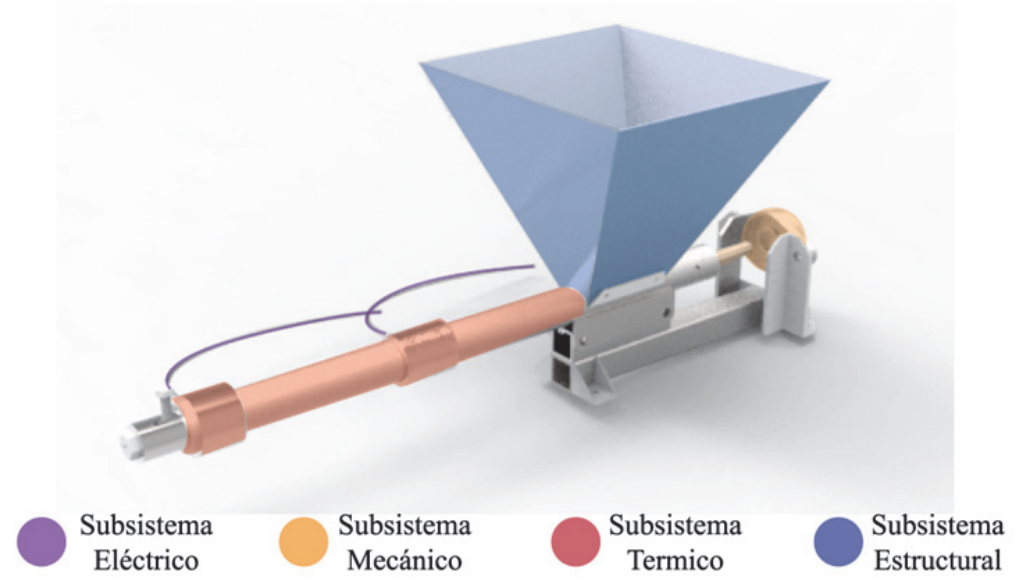

Fig.10 Identificación de los subsistemas de la máquina a partir de colores.

\section{Conclusiones}

Actualmente, en el mundo es necesario recapacitar sobre los problemas mayores que nos acogen, este trabajo aborda dos de ellos: la pobreza y la acumulación de residuos sólidos, en el caso particular el plástico. Ambos fenómenos desde una comunidad puntual en un país Latinoamericano, donde desde el diseño se aporta con soluciones centradas en tratamientos sistémicos y participativos, logrando soluciones impactantes en el contexto local con un alto nivel de apropiación. 
El uso de metodologías participativas aumentan la receptividad y la apropiación de los proyectos realizados para las comunidades, hacer uso de ellas requiere de disposición, de trabajo en equipo y de coordinación entre la comunidad y los diseñadores. Crear sincronía en el desarrollo de estas dinámicas hace que se genere confianza en los procesos y para que los resultados puedan realmente implementarse es necesario hacer acompañamiento y seguimiento.

El uso de las metodologías sistémicas permiten un abordaje íntegro del problema, escalando la visualización del mismo y logrando impactar mucho más en las actividades de reciclaje llevadas a cabo por la comunidad.

El diseño es una profesión que permite mejorar las condiciones de vida de las personas vulnerables, este pensamiento emerge en los años 70s y hoy en día hace parte de una de las líneas de investigación y desarrollo de este siglo.

\section{Agradecimientos}

A la comunidad de reciclaje Nashira, por permitir ser el caso de estudio para el desarrollo de este trabajo. Al SENA, Servicio Nacional de Aprendizaje por brindar el apoyo técnico para el manejo de los plásticos. A la Universidad Nacional de Colombia, como institución formadora de profesionales en diseño industrial.

\section{References}

ASSOCIATION OF PLASTICS MANUFACTURERS. (2015). Plastics - the facts 2015. An analysis of European plastics production, demand and waste data. Brussels: Plastics Europe.

BANCO INTERNACIONAL DE DESARROLLO - ASOCIACIÓN DE RECICLADORES DE BOGOTÁ. (2011). Resumen ejecutivo estudio nacional del reciclaje. Bogotá.

BERENGUER, JAIME. CORRALIZA, JOSE A. (2000) “preocupación ambiental y comportamientos ecológicos” en psicothema, Vol. 12, $\mathrm{N}^{\circ} 3$, pp 325-329.

CROSS, N. (2003). Metodos de diseño: estrategias para el diseño de productos. Editorial Limusa. Mexico D.F.

ELLEN MACARTHUR FUNDATION. (2016) The new plastics economy:rethinking the future of plastic.

HERNANDIS, B. IRIBARREN, E. (2000). Diseño de nuevos productos: una perspectiva sistémica. Valencia: Universidad Politécnica de Valencia.

HERNANDIS, B. (2015). Gestión de lo intangible para optimizar el diseño de lo tangible. Valencia: Universidad Politécnica de Valencia.

HERNANDIS, B. \& GUERRERO, M. (2014). "Estudio comparativo de las acciones a considerar en el proceso de diseño conceptual desde la ingeniería y el diseño de productos" en ingeniare, vol. 22 No 3, 2014, pp. 398-411.

PROGRAMA DE NACIONES UNIDAS. (2015). Objetivos de Desarrollo del Milenio informe 2015. Bogotá: PNUD.

RAMIREZ, T. LECUONA, M. CARDOZO, J. (2012) "diseño y bienestar humano: puntos de encuentro a partir de metodologías de diseño" en iconofacto. Vol. 8, $\mathrm{N}^{\circ} 10$, pp 88-114.

RODRÍGUEZ R. (1994).Teoría de sistemas y gestión de las organizaciones. Instituto andino de sistemas.

SANDERS, E. (2013) "Perspectives on Design in Participation" en Wer Gestaltet die Gestaltung? Praxis, Theorie und Geschichte des Partizipatorischen Designs", Mareis, C., Held, M. and Joost, G. Verlag. Transcript design.

SANDERS, E. \& STAPPERS, P. (2008). "Co-creation and the new landscapes of design". En CoDesign: International Journal of CoCreation in Design and the Arts Special Issue on Making. Vol. 4, N¹, pp 5-18. 
Recliclaje de plásticos de consumo masivo. Caso comunidad de reciclaje Nashira.

SANDERS, E. \& STAPPERS, P. (2014). "Probes, Toolkits and Prototypes: Three Approaches to Making in Codesigning". En CoDesign: International Journal of CoCreation in Design and the Arts Special Issue on Making. Vol. $10, \mathrm{~N}^{\circ} 1$, pp 5-14. 